\title{
Phosphodiesterase 2: anti-adrenergic friend or hypertrophic foe in heart disease?
}

\author{
Michael Wagner $^{1} \cdot$ Hind Mehel $^{2} \cdot$ Rodolphe Fischmeister $^{2} \cdot$ Ali El-Armouche ${ }^{1}$
}

Received: 17 August 2016 / Accepted: 17 August 2016/Published online: 24 August 2016

(C) Springer-Verlag Berlin Heidelberg 2016

Regardless of the underlying cause, heart failure (HF) patients typically show a hyperactive sympathetic nervous system with elevated plasma catecholamine levels and subsequent activation of the $\beta$-adrenoceptor ( $\beta$-AR) pathway (El-Armouche and Eschenhagen 2009). Chronically, this leads to attenuated cardiac $\beta$-AR responsiveness mainly as a result of a decrease in $\beta_{1}$-AR density, uncoupling from stimulatory $G$ proteins (Gs) through higher $\beta$-AR kinase ( $\beta$ ARK), deactivation of phosphatase inhibitor 1 , and higher levels of inhibitory $G$ proteins $\left(\mathrm{G}_{\mathrm{i}}\right)$ (Bristow et al. 1982; El-Armouche and Eschenhagen 2009; El-Armouche et al. 2008). In concert, these molecular alterations are assumed to be the basis for the blunted responses to catecholamines in the failing heart, a phenomenon called " $\beta$-AR desensitization." On the one hand, these changes may contribute to the progression of HF by further compromising the contractile performance of the failing heart. On the other hand, $\beta$-AR desensitization may protect from cardiotoxic $\beta$-AR stimulation, e.g., arrhythmias and myocardial hypertrophy. Accordingly, $\beta$-AR blockers are first-line recommended drugs in HF, whereas phosphodiesterase (PDE) inhibitors have been banned from chronic use due to increased mortality (El-Armouche and Eschenhagen 2009; Packer et al. 1991).

Recently, we have shed new light on an old but to date somewhat neglected player in cardiac $\beta$-AR signaling, PDE2, which is upregulated in failing hearts and fulfills

Ali El-Armouche

Ali.El-Armouche@tu-dresden.de

1 Institut für Pharmakologie und Toxikologie, Technische Universität Dresden, Fetscherstraße 74, 01307 Dresden, Germany

2 INSERM UMR-S 1180, Univ. Paris-Sud, Université Paris-Saclay, Châtenay-Malabry, France the criteria to be part of the $\beta$-AR desensitization machinery in HF (Hartzell and Fischmeister 1986; Mehel et al. 2013). PDEs degrade cyclic nucleotides, among others the second messenger cyclic adenosine monophosphate (cAMP) that is produced upon activation of $\beta$-ARs. Thereby, PDEs ensure temporal and spatial confinement of cAMP signals, resulting in distinct intracellular cAMP pools (Fischmeister et al. 2006). Intriguingly, PDE2 is stimulated up to 40 -fold by cGMP, establishing a potentially important cellular cross talk to the cardioprotective natriuretic peptide and NO systems (Fig. 1) (Jager et al. 2010). We have shown that PDE2 abundance, activity, and transcript levels are upregulated in human and animal models of HF (Mehel et al. 2013). Using the well-known chronical isoprenaline infusion model in rodents, we showed that pathological $\beta$-AR overstimulation alone is sufficient to induce higher PDE2 abundance and activity to a similar extent as observed in human end-stage HF, clearly indicating that PDE2 upregulation is a consequence of pathological $\beta$-AR overstimulation. Moreover, adenoviral overexpression of PDE2 dramatically blunted $\beta-A R$ inotropic responses, cellular hypertrophy, and arrhythmogenic events in isolated ventricular myocytes (Fig. 2) (Mehel et al. 2013). Taken together, greater PDE2 expression and activity in the failing heart is very likely a new player in the desensitization machinery of the $\beta$-AR signaling pathway. We postulated that this constitutes a potentially important ANP/BNP/cGMP-triggered defense mechanism during cardiac stress, in particular during excessive $\beta$-AR drive.

Recently, however, our concept of greater PDE2 as a protective mechanism in the failing heart has been challenged. Zoccarato et al. (2015) suggest in a number of well-designed experiments on isolated cardiomyocytes as well as in vivo that PDE2A regulates a local cAMP pool 


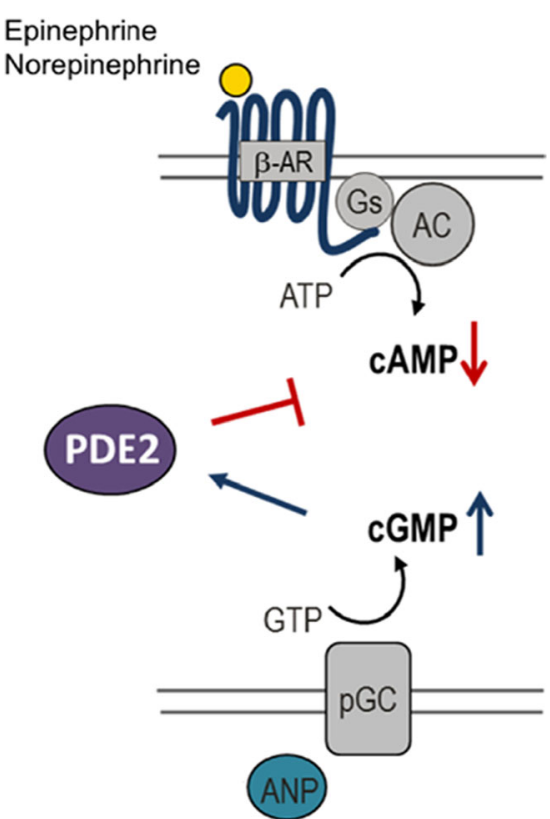

Fig. 1 PDE2 is a cGMP-stimulated phosphodiesterase. AC adenylyl cyclase, $A N P$ atrial natriuretic peptide, $p G C$ plasma membrane-bound guanylyl cyclase

that acts anti-hypertrophically by activating PKA II, leading to phosphorylation of nuclear factors of activated $\mathrm{T}$ cells (NFAT) and reducing their nuclear localization which reduces the expression of hypertrophic genes.
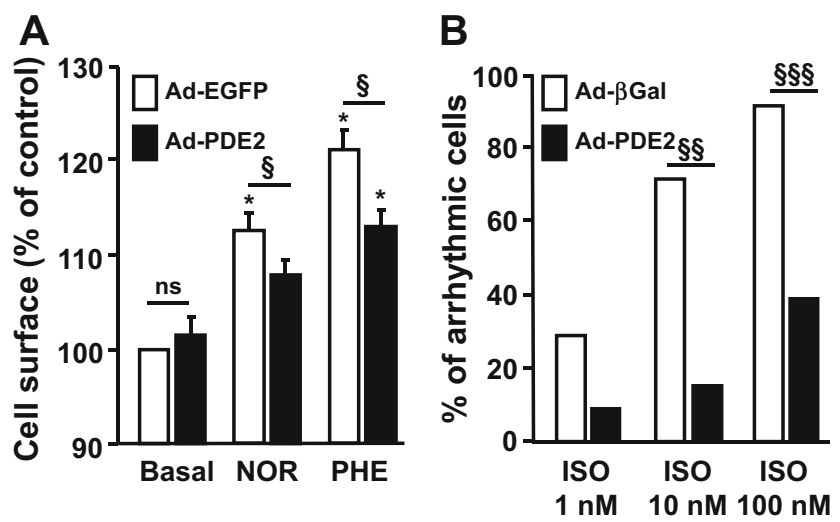

Fig. 2 a Adult rat ventricular cardiomyocytes (ARVMs) were stimulated with $10 \mu \mathrm{M}$ norepinephrine $(N O R)$ or $10 \mu \mathrm{M}$ phenylephrine $(P H E)$ at $37^{\circ} \mathrm{C}$ for $24 \mathrm{~h}$, and the cellular surface area was determined by microscopy (Mehel et al. 2013). $N=5-6$ preparations. b Percent of myocytes with spontaneous $\mathrm{Ca}^{2+}$ waves (indicated as arrhythmic cells) in the presence of increasing concentrations of isoprenaline (ISO). Intracellular $\mathrm{Ca}^{2+}$ was measured in ARVMs loaded with Fura-2 and electrically stimulated at a frequency of $0.5 \mathrm{~Hz} . N=6$ animals/condition with a total of 14-22 cells in each group (own unpublished data). ${ }^{*} p<0.05$ versus basal; $\S_{p}<0.05 ;{ }^{\S} p<0.01 ;{ }^{\S \S} p<0.001$, adenovirus encoding enhanced green fluorescent protein $(A d-E G F P)$ in a or adenovirus encoding $\beta$ galactosidase ( $A d-\beta G a l)$ in $\mathbf{b}$ versus adenovirus encoding PDE2A2 ( $A d-$ $P D E 2)$. ns not significant
Thus, not PDE2A activity but its inhibition would act anti-hypertrophically. These findings are hard to reconcile with our own previous findings. Zoccarato et al. suggest a remarkable approach to resolve this issue: In both publications, adenoviral overexpression of PDE2A was used for a crucial experiment to assess its effect on cell surface area in isolated cardiomyocytes. We found that PDE2 overexpression inhibits hypertrophy induced by both norepinephrine and phenylephrine (Fig. 7 of Mehel et al. 2013) in adult rat ventricular myocytes (ARVMs), while Zoccarato et al. suggest that PDE2A overexpression alone leads to hypertrophy in ARVMs (online Fig. III of Zoccarato et al. 2015) and does not attenuate hypertrophy induced by norepinephrine (online Fig. VII of Zoccarato et al. 2015). Since in our original publication, we normalized both the control virus and the PDE2A overexpressing virus under control conditions to $100 \%$, Zoccarato et al. suggest that our results could have been obfuscated by the normalization. Moreover, when they normalize their data, it looks similar to ours (online Fig. XII of Zoccarato et al. 2015). Consequently, they imply that we may have misinterpreted our data. Fortunately, this is not the case. To demonstrate this, we include the non-normalized data as Fig. 2a. The figure clearly demonstrates that in our hands, PDE2A2 overexpression in itself does not lead to hypertrophy in ARVMs but prevents norepinephrineinduced hypertrophy and significantly attenuates phenylephrine-induced hypertrophy. Hence, other factors must account for the different results and conclusions. Regarding the adenovirus experiments, differences in the construct, the transfected PDE2 isoform, and the cellular localization of the transfected PDE2 are possible explanations. Regarding the overall results, the conflicting evidence suggests that dependent on isoform, localization, and cellular and pathophysiological context, PDE2A may have protective anti-hypertrophic and antiarrhythmic as well as detrimental pro-hypertrophic effects in the cardiomyocyte. This should encourage researchers in this field to explore the pathophysiological roles of PDE2 in models of heart disease as well as to look more closely at its action on distinct cellular cAMP (and cGMP) pools. PDE2 inhibitors and maybe future activators will be helpful to assess overall effects, but ultimately, transgenic approaches will be necessary to assess the effect in specific cell types and subcellular locations. Consequently, we expect the present controversy on the cardiac actions of PDE2 to stimulate and accelerate the emerging field of PDE2-related research and to help design better and more focused hypotheses. Cardiomyocytespecific PDE2 transgenic and KO mice are currently in the pipeline, and their phenotyping in state-of-the-art models of heart disease will help to clarify whether PDE2 is a friend or a foe in heart disease. 
Acknowledgments The authors are supported by the Deutsche Forschungsgemeinschaft (WA 2586/4-1 to MW and EL 270/7-1 to AEA), the Fondation pour la Recherche Médicale (to HM and RF), and the Investment for the Future program ANR-11-IDEX-0003-01 within the LABEX ANR-10-LABX-0033 (to RF).

\section{References}

Bristow MR et al. (1982) Decreased catecholamine sensitivity and betaadrenergic-receptor density in failing human hearts. N Engl J Med 307:205-211. doi:10.1056/NEJM198207223070401

El-Armouche A, Eschenhagen T (2009) Beta-adrenergic stimulation and myocardial function in the failing heart. Heart Fail Rev 14:225-241. doi:10.1007/s10741-008-9132-8

El-Armouche A et al. (2008) Phosphatase inhibitor-1-deficient mice are protected from catecholamine-induced arrhythmias and myocardial hypertrophy. Cardiovasc Res 80:396-406. doi:10.1093/cvr/cvn208
Fischmeister R, Castro LR, Abi-Gerges A, Rochais F, Jurevicius J, Leroy J, Vandecasteele G (2006) Compartmentation of cyclic nucleotide signaling in the heart: the role of cyclic nucleotide phosphodiesterases. Circ Res 99:816-828. doi:10.1161/01.RES.0000246118.98832.04

Hartzell HC, Fischmeister R (1986) Opposite effects of cyclic GMP and cyclic AMP on Ca2+ current in single heart cells. Nature 323:273275. doi:10.1038/323273a0

Jager R, Schwede F, Genieser HG, Koesling D, Russwurm M (2010) Activation of PDE2 and PDE5 by specific GAF ligands: delayed activation of PDE5. Br J Pharmacol 161:1645-1660. doi:10.1111/j.1476-5381.2010.00977.x

Mehel H et al. (2013) Phosphodiesterase-2 is up-regulated in human failing hearts and blunts beta-adrenergic responses in cardiomyocytes. J Am CollCardiol 62:1596-1606

Packer M et al. (1991) Effect of oral milrinone on mortality in severe chronic heart failure. The PROMISE Study Research Group. N Engl J Med 325:1468-1475. doi:10.1056/NEJM199111213252103

Zoccarato A et al. (2015) Cardiac hypertrophy is inhibited by a local pool of cAMP regulated by phosphodiesterase 2. Circ Res 117:707-719. doi:10.1161/CIRCRESAHA.114.305892 